\section{Integrating Neuropsychological Outcomes into Clinical Studies for Treating Secondary or Primary Intracranial Malignancies in the Era of Modern Radiation Therapy}

Received: June 27, 2019; Accepted: July 17, 2019; Published: July 18, 2019

\section{Introduction}

In managing patients with brain metastasis in the disciplines of neuro-oncology, neurosurgery, and radiation oncology, intracranial control and care [1-3]. Of note, the controversies and problems associated with delayed radiation-related cognitive dysfunction cannot be ignored, particularly in patients who have undergone whole-brain radiation therapy (WBRT) $[4,5]$. Concerning neurocognitive consequences related to cranial irradiation, previous studies have shown that impaired hippocampal neurogenesis [6,7] may be responsible for such cognitive impacts resulting from the delivery of WBRT. Therefore, it has been hypothesized that conformal hippocampal sparing during the course of WBRT (HS-WBRT) [8,9] would contribute to meaningful preservation with regard to neurocognitive functions (NCFs).

Herein we would like to explicitly mention our pervious publications and ongoing research work. First, a preliminary report published in a local academic journal affiliated with our institute documented that incorporating objective neurobehavioral evaluations greatly enhances our understanding of neurocognitive functional outcomes when treating patients with oligometastatic brain disease. In addition, it was disclosed that relevant NCFs would be preserved through the delivery of HS-WBRT. Second, according to our preliminary and robust findings regarding the correlations between various hippocampal dosimetric parameters and relevant NCFs of interest, particularly hippocampus-dominated memory functions [10], a dose-effect relationship was observed; specifically, the dosimetric variables concerning left-side hippocampus were significantly associated with the status of verbal memory function. Definitely a deeper and more clarified understanding of the correlations between hippocampal dosimetry and neuropsychological outcomes would guide us when formulating and proposing future study protocols $[11,12]$.

In addition to our first prospective observational study (HSWBRT clinical study) essentially integrating neuropsychological
Yn Lin S ${ }^{1,2 *}$, Tsan DL ${ }^{3,4}$, Chuang $\mathrm{CC}^{4,5}$, Pai $\mathrm{PC}^{1,2}$, Chiang $\mathrm{YY}^{1}$, Yang $\mathrm{CC}^{6}$, Lu YJ ${ }^{4,5}$, Huang YC $\mathrm{YC}^{4,5}$ and Chou WC ${ }^{4,7 *}$

1 Department of Radiation Oncology, Chang Gung Memorial Hospital and Chang Gung University, Taoyuan, Taiwan

2 Department of Medical Imaging and Radiological Sciences, College of Medicine, Chang Gung University, Taoyuan, Taiwan

3 Department of Radiation Oncology, Chang Gung Memorial Hospital, Keelung, Taiwan

4 College of Medicine, Chang Gung University, Taoyuan, Taiwan

5 Department of Neurosurgery, Chang Gung Memorial Hospital, Taoyuan, Taiwan

6 Department of Psychology, National ChengChi University, Taipei, Taiwan

7 Department of Hematology-Oncology, Chang Gung Memorial Hospital and Chang Gung University, Taoyuan, Taiwan

Corresponding authors: Shinn-Yn Lin

”rt3126@gmail.com

Department of Radiation Oncology, Proton and Radiation Therapy Center, Chang Gung Memorial Hospital and Chang Gung University, Taoyuan, Taiwan.

Tel: +886-3-3281200 ext: 7000

Citation: Lin SY, Tsan DL, Chuang CC, Pai PC, Chiang YY, et al. (2019) Integrating Neuropsychological Outcomes into Clinical Studies for Treating Secondary or Primary Intracranial Malignancies in the Era of Modern Radiation Therapy. Neurooncol Open Access Vol.4 No.1: 4. 
elaborate further the subsequent study protocols in several directions. First of all, for patients with oligometastatic brain disease who still have intracranial gross metastatic lesion(s), the planning technique of simultaneously integrated boost (SIB) had better be incorporated into the course of HS-WBRT [13] in order to accomplish both improved intracranial local control and preserved neurocognitive functional outcomes. Second, in contrast to the clinical setting tailored to the above HS-WBRT plus SIB study, our research team has also started to aims at exploring the role of prophylactic cranial irradiation ( $\mathrm{PCl}$ ) via the technique of HS-WBRT in managing patients with pathologically nodal positive non-small-cell lung cancer (NSCLC) who have just undergone curative thoracic surgery for treating newly-diagnosed primary lung cancer [14]. As shown in Table 1, the scope of our neurocognitive research has been extended to investigate other disease entities such as primary CNS lymphoma and infiltrative gliomas requiring postoperative adjuvant RT. Besides, almost all study protocols listed in Table 1 were prospective observational studies except for the PCl study via HS-WBRT for postoperative patients with nodal positive NSCLC.

As shown in the above table listing the study protocols conducted by our research team, the second different and distinct disease entity our research has been focusing on is newly-diagnosed primary central nervous system lymphoma (PCNSL) [15]. Thanks to the multidisciplinary management combining neurooncology, neurosurgery, radiation oncology, hematology, and even neurobehavioral sciences, undoubtedly intracranial control and survival outcomes have been improving under multidisciplinary cancer care. Nevertheless, previous evidence has shown that delayed treatment-related cognitive sequelae emerged as a significant debilitating complication of combined modality treatment encompassing whole-brain irradiation in treating PCNSL patients, especially when effective treatment can eventually achieve better disease control and even prolong survival profiles. Thus, we have originally proposed and carried out a prospective cohort study with a longitudinal assessment of neuropsychological/neurocognitive functions, neuroimaging, and quality of life for newly-diagnosed PCNSL patients who would be treated with cranial radiotherapy combined with/without methotrexate (MTX)-based chemotherapy on the basis of multidisciplinary treatment guidelines actually and consistently implemented at our institute.

Although RT-associated techniques employed in delivering WBRT might not be the key research question addressed in the above study, actually whole brain irradiation delivered in this PCNSL study mainly relied on a conformal treatment planning rather than conventional WBRT via simply laterally opposed fields. As a consequence, a highly conformal 3-dimensional treatment planning with an additional planning technique of simultaneously integrated boost (SIB) must have attained optimized dose distributions and contributed to a significantly more favourable intracranial disease control and even better survival outcomes in this era of contemporary and modern radiotherapy.

In the real-world clinical practice at our institute, it has been acknowledged that the administration of induction therapy with "high-dose" MTX was almost not feasible even given to younger patients because there were unsatisfactory morbidity rates resulting from high-dose MTX at our institute. As a consequence, the majority of newly diagnosed PCNSL patients we encountered have been managed with combined chemoradiation in a sandwiched fashion in which a conventional dosage $\left(1 \mathrm{~g}\right.$ per $\left.\mathrm{m}^{2}\right)$ of intravenous MTX was prescribed for the past two decades.

From the viewpoint of radiation oncologists based on radiological rationales, there have been several previous prospective studies conducted by Radiation Therapy Oncology Group (RTOG) proposing the scheme of hyper fractionated WBRT (hWBRT) instead of a course of conventionally fractionated WBRT in order to alleviate the probability and severity of potential WBRT-induced neuropsychological consequences $[16,17]$. Theoretically and reasonably, delivering a hyperfractionated scheme of WBRT via 3-D conformal treatment planning with an additional SIB technique (if needed) will achieve both optimized intracranial (lymphoma) control and diminished WBRT-related neurocognitive impacts. Therefore, a prospective cohort study has just been proposed and under review for being funded by our institutional research foundation. Furthermore, by administering objective multi-domain neurocognitive and neuropsychological assessments, the status and change in NCFs before and after the course of hyperfractionated conformal WBRT will be examined and analysed.

Regarding our study protocols addressing neuropsychological and oncological outcomes in chronological order, the third unique and different disease entity under ongoing investigation is radiationnaive malignant glioma excluding pathological grade IV disease; namely, a prospective follow-up study has been carried out in grade 2 or 3 glioma patients indicated for receiving postoperative modern radiotherapy. This prospective observational study is anticipated to examine thoroughly neurocognitive impacts of postoperative adjuvant RT with using modern radiotherapeutic techniques on patients' neurocognitive performances and verify that functional preservation might be achieved by sparing the contralateral hippocampus. Meanwhile, adjuvant partial brain irradiation delivered in these glioma patients will contribute to satisfactory progress-free survival interval, attaining the international level.

In our opinions, integrating functional outcomes including NCF assessments with commonly applied neuro-oncological outcomes would help neuro-oncologists and radiation oncologists manage their secondary or primary intracranial malignancies in the era of modern radiation therapy. For instance, when treating cancer patients harboring brain metastases, particularly those with oligometastatic brain disease and a satisfactory/fair performance status, the delivery of HS-WBRT course indeed has achieved both functional preservation and satisfactory intracranial control by restricting the dose irradiating the hippocampus during the WBRT course. Besides, regarding postoperative adjuvant partial cranial irradiation employed in treating patients with newly-diagnosed 
Table 1: Protocol registration of our neuropsychological studies on clinicaltrials.gov.

\begin{abstract}
Title
A Prospective Study of the Impact
of Hippocampal Avoidance During Whole Brain Radiotherapy on Neurocognitive Function Decline Outcomes Follow-up in Primary CNS Lymphoma Patients Treated With Cranial Radiotherapy Combined With or Without MTXbased Chemotherapy According to the Multidisciplinary Treatment Guidelines Implemented at a Single Institute
\end{abstract}

Neurobehavioral Outcomes and Quality of Life in Pediatric Patients With Brain or Head/Neck Tumors Receiving Proton or Photon Radiotherapy

Hippocampal-Sparing Prophylactic Cranial Irradiation in Pathologically Nodal Positive NonSmall-Cell Lung Cancer

Neurocognitive Impact and Dose-Effect Relationship of Hippocampal Avoidance During Whole Brain Radiotherapy Plus Simultaneous Integrated Boost - A Prospective Follow-up Study

Neuropsychological and Oncological Outcomes in Grade 2 or 3 Glioma Patients Undergoing Postoperative Modern Radiotherapy

Prospective Neurobehavioral Functions in Newly-diagnosed Patients With Primary CNS Lymphoma Treated With Hyperfractionated Conformal Whole-brain Radiation Therapy Plus Simultaneous Integrated Boost

Patients

Intervention

Outcomes

Website

Patients with oligometastatic

brain disease (i.e., the number and extent of brain metastatic lesions should be no more than three metastatic foci with a greatest

diameter no more than $4 \mathrm{~cm}$ )

Patients with lung cancer referred

for arranging prophylactic cranial irradiation

Newly-diagnosed patients with primary CNS Iymphoma

A conventional treatment protocol with courses of combined chemoradiation in a sandwiched manner at our institute

A course of whole-brain radiation (WBRT) using the technique of hippocampal sparing during the course of WBRT (HS-WBRT)

Primary endpoint: delayed recall, as determined by the change in verba and non-verbal memory functions from the baseline assessment to 4 months after the start of HS-WBRT.

\section{Neuropsychological outcome} measures involve four domains sensitive to disease and treatment effects including executive function and verbal memory; the change in these domains are acquired from baseline to 4 months after completing all courses of chemoradiation is our primary endpoint.
Newly-diagnosed pediatric/ adolescent patients with brain tumors or head/neck cancers

Adult patients with NSCLC have received definitive surgery and then then the presence of pathologically nodal positive disease makes the patient

referred to undergo the subsequent adjuvant platinumbased radiotherapy.

Patients with oligometastatic brain disease (i.e., the number and extent of brain metastatic lesions should be no more than three metastatic foci with a greatest diameter no more than $3 \mathrm{~cm}$

Patients with infiltrative gliomas excluding the presence of GBM who have received craniotomy plus tumor resection or at least biopsy with pathologic conformation

Newly-diagnosed patients with primary CNS lymphoma
A course of HS-WBRT plus simultaneous integrated boost (SIB)

Postoperative adjuvant

$\mathrm{RT}$ is indicated in this prospective observational study. Prescription dose will be $5000-6000$ cGy in 25 - 30 fractions during 5 7 weeks.

Each freshly-diagnosed patient with primary treated with combined chemoradiation in a sandwiched way, in which 2 cycles of induction chemotherapy with intravenous methotrexate (MTX in a conventional dose of $1 \mathrm{~g} / \mathrm{m}^{2}$ ) and intrathecal MTX are followed by a complete course of hyper fractionated WBRT* and then high-dose cytarabine for 2 cycles. CNS lymphoma will be

\section{NCT02608762}

The time to the development of brain metastasis/metastases (BM), irrespective of the absence of active neurological symptoms

The primary outcome measure is delayed recall, as determined by the change in verbal and non-verbal memory functions from the baseline assessment to 4 months after the start of HS-WBRT.

The primary outcome measure is delayed recall, as determined by the change in verbal memory or nonverbal memory from the baseline assessment to 4 months after the start of postoperative adjuvant RT.

The primary outcome measure is the change in patients' capability of information processing indicated by the Paced Auditory Serial Addition Test - Revised (PASAT-R), from the baseline before undergoing the WBRT course to the timing after going through the entire courses of combined chemoradiation.
Under protocol review by

ClinicalTrials. gov 
or RT-naïve infiltrative glioma, it is highly recommended to spare the contralateral hippocampus of being unnecessarily irradiate, if technically feasible, in an attempt to diminish the extent of neurocognitive impacts related to the contralateral hippocampus, which is significantly associated with verbal or non-verbal memory functions. Last but not least, even for treating newly-diagnosed primary CNS lymphoma patients at our institute, multi-domain NCFs indeed become stabilized and generally improved after the

\section{References}

1 Maclean J, Fersht N, Singhera M, Mulholland P, McKee O, et al. (2013) Multi-disciplinary management for patients with oligometastases to the brain: results of a 5 year cohort study. Radiat Oncol 8.

2 Owonikoko TK, Arbiser J, Zelnak A, Shu HKG, Shim H, et al. (2014) Current approaches to the treatment of metastatic brain tumours. Nat Rev Clin Oncol 11: 203-222.

3 Scoccianti S, Ricardi U (2012) Treatment of brain metastases: Review of phase III randomized controlled trials. Radiother Oncol 102: 168179 .

4 Soffietti R, Kocher M, Abacioglu UM, Villa S, Fauchon F, et al. (2013) A European Organisation for Research and Treatment of Cancer Phase III Trial of Adjuvant Whole-Brain Radiotherapy Versus Observation in Patients With One to Three Brain Metastases From Solid Tumors After Surgical Resection or Radiosurgery: Quality-of-Life Results. J Clin Oncol 31: 65-72.

5 Aoyama H, Tago M, Kato N, Toyoda T, Kenjyo M, et al. (2007) Neurocognitive function of patients with brain metastasis who received either whole brain radiotherapy plus stereotactic radiosurgery or radiosurgery alone. Int J Radiat Oncol Biol Phys 68: 1388-1395.

6 Rola R, Raber J, Rizk A, Otsuka S, VandenBerg SR, et al. (2004) Radiation-induced impairment of hippocampal neurogenesis is associated with cognitive deficits in young mice. Exp Neurol 188: 316-330.

7 Byrne TN (2005) Cognitive sequelae of brain tumor treatment. Curr Opin Neurol 18: 662-666.

8 Gondi V, Pugh SL, Tome WA, Caine C, Corn B, et al. (2014) Preservation of memory with conformal avoidance of the hippocampal neural stem-cell compartment during whole-brain radiotherapy for brain metastases (RTOG 0933): a phase II multi-institutional trial. J Clin Oncol 32: 3810-3816.

9 Lin SY, Yang CC, Wu YM, Tseng CK, Wei KC, et al. (2015) Evaluating the impact of hippocampal sparing during whole brain radiotherapy course of conformal WBRT combined with methotrexate-based chemotherapy is completed.

\section{Funding}

This article-related study was principally funded by Chang Gung Memorial Hospital at Keelung, Taiwan, R.O.C. (grant number CMRPG2G0472) and Chang Gung Memorial Hospital at Taoyuan, Taiwan, R. O. C. (grant number CMRPG3J0101).

on neurocognitive functions: A preliminary report of a prospective phase II study. Biomed J 38: 439-449.

10 Zhao R, Kong W, Shang J, Zhe H, Wang YY (2017) HippocampalSparing Whole-Brain Radiotherapy for Lung Cancer. Clin Lung Cancer 18: 127-131.

11 Tsai PF, Yang CC, Chuang CC, Huang TY, Wu YM, et al. (2015) Hippocampal dosimetry correlates with the change in neurocognitive function after hippocampal sparing during whole brain radiotherapy: a prospective study. Radiat Oncol 10: 1-15.

12 Ma TM, Grimm J, Mclntyre R, Anderson Keightly H, Kleinberg LR, et al. (2017) A prospective evaluation of hippocampal radiation dose volume effects and memory deficits following cranial irradiation. Radiother Oncol 125: 234-240.

13 Shinn-Yn Lin, Chi-Cheng Chuang (2016) Neurocognitive Impact and Dose-Effect Relationship of Hippocampal Avoidance During Whole Brain Radiotherapy Plus Simultaneous Integrated Boost - A Prospective Follow-up Study.

14 Shinn-Yn Lin, Chi-Cheng Chuang (2015) Hippocampal-Sparing Prophylactic Cranial Irradiation in Pathologically Nodal Positive NonSmall-Cell Lung Cancer.

15 Shinn-Yn Lin, Chi-Cheng Chuang (2015) Prospective Neurobehavioral Outcomes Follow-up in Primary CNS Lymphoma Patients Treated With Cranial Radiotherapy Combined With or Without MTX-based Chemotherapy According to the Multidisciplinary Treatment Guidelines Implemented at a Single Institute.

16 Fisher B, Seiferheld W, Schultz C, DeAngelis L, Nelson D, et al. (2005) Secondary analysis of Radiation Therapy Oncology Group study (RTOG) 9310: an intergroup phase II combined modality treatment of primary central nervous system lymphoma. J Neurooncol 74: 201-205.

17 Glass J, Won M, Schultz CJ, Brat D, Bartlett NL, et al. (2016) [NRG Oncology RTOG 0227] Phase I and II Study of Induction Chemotherapy With Methotrexate, Rituximab, and Temozolomide, Followed By Whole-Brain Radiotherapy and Post irradiation Temozolomide for Primary CNS Lymphoma: NRG Oncology RTOG 0227. J Clin Oncol 34 1620-1625. 